\title{
HLA class I and II, interferon, interleukin 2, and the interleukin 2 receptor expression on labial biopsy specimens from patients with Sjögren's syndrome
}

\author{
DEBORAH ROWE, MERYL GRIFFITHS, JANE STEWART, \\ DANIELLA NOVICK, P C L BEVERLEY, AND D A ISENBERG
}

From the ICRF Human Tumour Immunology Group, the Department of Morbid Anatomy and the Department of Oral Surgery, School of Medicine, University College, London; the Department of Virology, Weizman Institute, Rehovot, Israel; and the Bloomsbury Rheumatology Department, Rheumatologyo Research Department, University College and the Middlesex Hospital Medical School, London

SUMMARY Labial biopsy specimens from eight patients with primary Sjögren's syndrome (SS), $\stackrel{\circ}{\rightarrow}$ 10 patients with secondary SS, and three healthy controls were studied with monoclonal? antibodies identifying HLA class I and class II antigens; interferon- $\alpha, \beta$, and $\gamma$; interleukin 20 (IL2); and the IL2 receptor (Tac) among others. In the normal biopsy specimens there was evidence of HLA class I and, to a lesser extent, class II antigens in both ducts and acini, though $\vec{\infty}$ this was much less marked than in the Sjögren's biopsy specimens. Interferon- $\gamma$ staining, but not. ${ }^{-}$ interferon- $\alpha$ or $\beta$, was also considerably enhanced in the biopsy specimens from the patients with Sjögren's syndrome. These data support the view that in Sjögren's syndrome the release of interferon- $\gamma$ may be involved in the induction of class II determinants. Our observations wereo broadly similar in both primary and secondary Sjögren's syndrome except that patients witho primary Sjögren's syndrome tended to have more diffusely scattered T lymphocytes.

Sjögren's syndrome is an autoimmune disorder characterised histologically by a primarily lymphocytic infiltration of the salivary and lachrymal glands. Plasma cells are also commonly found in the cellular infiltrate which accompanies the coincident destruction of the acinar tissue and proliferation of the ducts. Most patients with Sjögren's syndrome may be divided into those with primary disease, dry eyes and mouth alone, and those with secondary disease, in whom these symptoms are set against a background of another autoimmune rheumatic disease such as systemic lupus erythematosus or rheumatoid arthritis.

In a previous study of labial biopsy specimens from patients with secondary Sjögren's syndrome we showed that most of the infiltrating cells were T lymphocytes, among which those expressing the helper/inducer phenotype predominated. ${ }^{1}$ Many of

Accepted for publication 17 March 1987.

Correspondence to Dr D A Isenberg. Bloomsbury Rheumatology Department. Arthur Stanley House. Tottenham Street. London W1P 9PG. these cells appeared to co-express HLA class IP antigens, suggesting that these $T$ lymphocytes were activated. These observations have been? confirmed, ${ }^{2}$ and reports suggest that the sames. findings are evident among patients with primary Sjögren's syndrome..$^{23}$

We have undertaken the present study to examing in more detail the relation between HLA class I and II antigens on both the glandular structures and th infiltrating cells, and interferon and interleukir expression. In particular we were interested to know whether our observations in muscle tissue from patients with polymyositis would be repeated in this related context. ${ }^{4-6}$ These observations included the demonstration that normal muscle does not express HLA class I antigens but that in damaged muscle of in fibres adjacent to an inflammatory infiltrate there is abundant HLA class I expression, which is closely matched to the detection of interferon- $\alpha, \beta$, and $\gamma_{\overline{0}}$ We have suggested that after some initial damag $\Phi^{+}$ the release of interferons from the infiltrating cells if responsible for the HLA class I expression, whic may help to drive the autoimmune process. 
Table 1 Patients with primary Sjögren's syndrome

\begin{tabular}{lllll}
\hline $\begin{array}{l}\text { Patient } \\
\text { No }\end{array}$ & Age & Sex & Ethnic origin & Treatment at time of biopsy \\
\hline 1 & 73 & M & $\begin{array}{l}\text { Caucasian } \\
\text { Indian }\end{array}$ & $\begin{array}{l}\text { Navidrex-K } 2 \mathrm{tab} / \text { day } \\
\text { Naprosyn 1 g/day, } \\
\text { Plaquenil } 400 \mathrm{mg} / \text { day }\end{array}$ \\
2 & 49 & M & & - \\
3 & 58 & F & Caucasian & - \\
4 & 61 & F & Caucasian & - \\
5 & 68 & F & Caucasian & Navidrex-K 2 tab/day \\
6 & 56 & F & Caucasian & Indocid 75 mg/day \\
7 & 51 & F & Caucasian & - \\
8 & 50 & F & Caucasian & - \\
\hline
\end{tabular}

Patients, materials, and methods

\section{PATIENTS}

Eight labial biopsy specimens from patients with primary Sjögren's syndrome and 10 from patients with secondary Sjögren's syndrome were studied. The details of these patients are shown in Tables 1 and 2. In addition, three patients attending University College Dental Hospital to have wisdom teeth removed agreed to have labial biopsies having signed a written consent approved by the hospital ethical committee.

\section{MATERIALS AND METHODS}

Frozen tissue sections $(6 \mu \mathrm{m})$ from patients with Sjögren's syndrome and from normal individuals were stained with a panel of monoclonal antibodies (MoAb). The monoclonal antibodies used were: $(a)$ 2A1: an IgG1 mouse MoAb. It identifies a nonpolymorphic determinant of HLA class I antigens; (b) UCHT1: an IgG1 mouse MoAb against the CD3 (T3) antigen present on mature $\mathrm{T}$ lymphocytes and some thymocytes; (c) DA2: an IgG1 MoAb with specificity for a non-polymorphic determinant of HLA class II antigens ${ }^{7}$; (d) 74-3: an IgG1 mouse antibody which binds interferon- $\alpha^{8} ;(e)$ 117-1: an IgG1 mouse MoAb which binds interferon- $\beta^{9} ;(f)$ 166-5: an IgG1 mouse MoAb which also binds interferon- $\gamma^{10} ;(g)$ DMS2: a MoAb to interleukin 2 (gift of Kendal A Smith); (h) DMS3: a MoAb to interleukin 2 (gift of Kendal A Smith); (i) anti-Tac: MoAb to the IL2 receptor ${ }^{11}$ (gift of T Waldmann).

The method used was as follows: $(a)$ unfixed sections were washed for two minutes in phosphate buffered saline (PBS) and incubated with the first antibody for one hour. (b) They were then incubated in R-anti M Ig (RAM) (concentrations determined by titration, usually around $1 / 100$ in PBS) containing $1 / 25$ pooled normal human serum for $30 \mathrm{~min}$. (c) The sections were then washed in running PBS for $30 \mathrm{~s}$ and then for $2 \mathrm{~min}$ in a trough. $(d)$ They were incubated for $30 \mathrm{~min}$ in an alkaline phosphatase/monoclonal anti alkaline phosphatase complex (APAAP). (e) Sections were again washed in running PBS for $30 \mathrm{~s}$ and then for $2 \mathrm{~min}$ in a trough. $(f)$ Steps $a-e$ were repeated three times, with incubation in RAM and APAAP reduced to $10 \mathrm{~min}$ each. $(g)$ The sections were then incubated in a fast red/naphthol AS-B1 phosphate substrate (Sigma Chemical Co, St Louis, MO, USA) containing 200 $\mu \mathrm{l}$ of $1 \mathrm{mM}$ levamisole per $10 \mathrm{ml}$ substrate solution. The substrate was dissolved in veronal acetate buffer $\mathrm{pH} 9 \cdot 2$. (h) Sections were washed in distilled water for 2 min. (i) Sections were counterstained in haematoxylin and mounted in Apathy's mounting medium. $(j)$ The biopsy specimens were all assessed 'blind' using a semiquantitative 0 to +++ scale. It has been shown that the APAAP method can detect antigens not seen by more

Table 2 Patients with secondary Sjögren's syndrome

\begin{tabular}{|c|c|c|c|c|c|}
\hline $\begin{array}{l}\text { Patient } \\
\text { No }\end{array}$ & $\begin{array}{l}\text { Accompanying } \\
\text { disease }\end{array}$ & Age & Sex & Ethnic origin & $\begin{array}{l}\text { Treatment at time } \\
\text { of biopsy ( } \mathrm{mg} / \text { day })\end{array}$ \\
\hline 9 & SLE & 34 & $\mathbf{F}$ & Caucasian & Dapsone 50 \\
\hline 10 & SLE & 41 & $\mathbf{F}$ & Caucasian & $\begin{array}{l}\text { Plaquenil } 400, \\
\text { Naprosyn } 500\end{array}$ \\
\hline 11 & SLE & 40 & $\mathbf{F}$ & Caucasian & Plaquenil 400 \\
\hline 12 & SLE & 68 & $\mathbf{F}$ & Caucasian & Feldene 20 \\
\hline 14 & SLE & 32 & $\mathrm{~F}$ & Caucasian & Plaquenil 400 \\
\hline 15 & SLE & 57 & $\mathbf{F}$ & Caucasian & Prednisolone 7.5 \\
\hline 16 & SLE & 31 & $\mathbf{F}$ & Chinese & - \\
\hline 17 & RA & 56 & $\mathbf{F}$ & Caucasian & $\begin{array}{l}\text { Plaquenil } 400 \text {, } \\
\text { Lederfen } 900\end{array}$ \\
\hline 18 & Polymyositis & 32 & $\mathbf{F}$ & Indian & $\begin{array}{l}\text { Prednisolone } 7 \cdot 5 \text {, } \\
\text { azathioprine } 100\end{array}$ \\
\hline
\end{tabular}




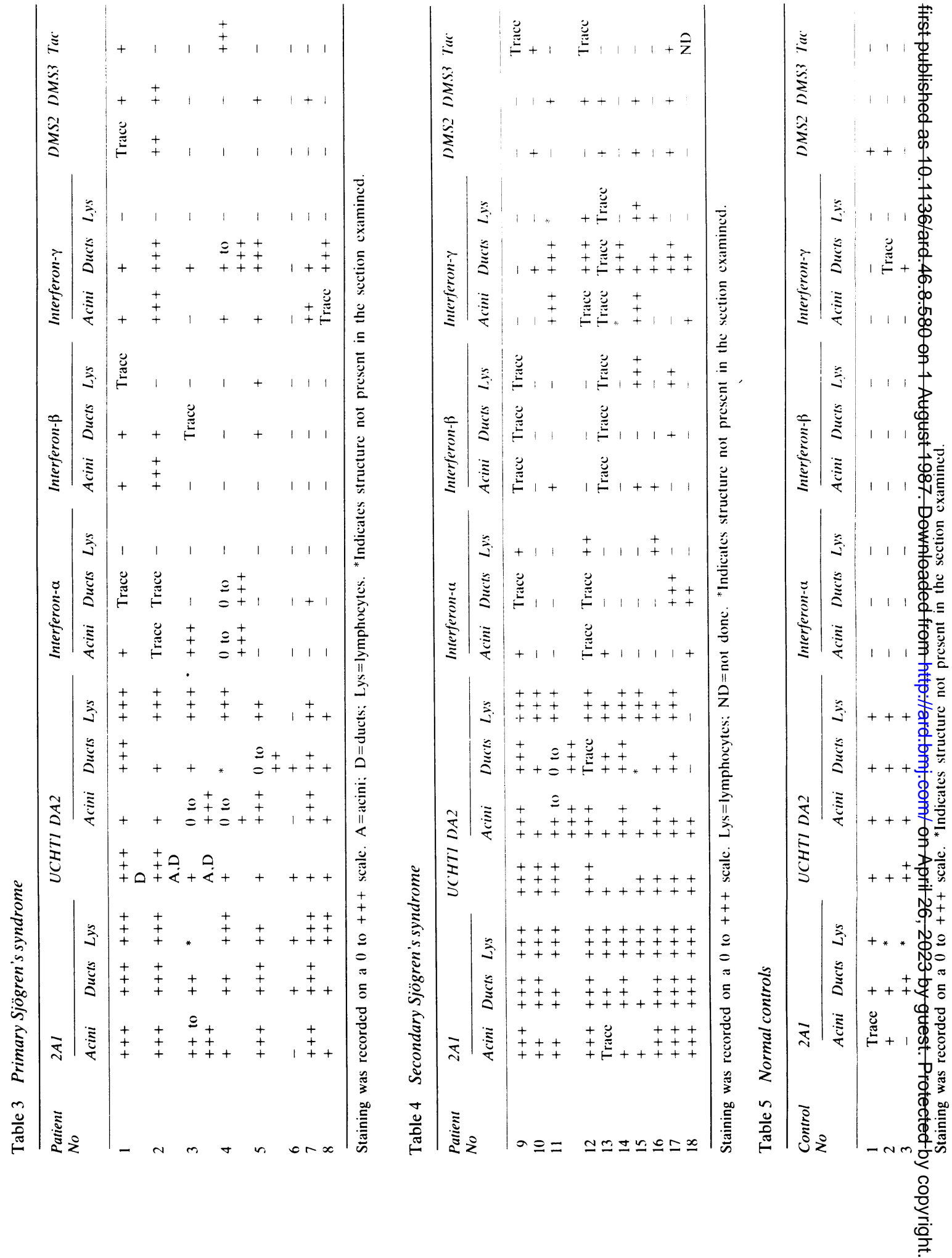


conventional methods when multiple layers of APAAP complexes are used. 5

\section{Results}

The results using the nine monoclonal antibodies are shown in Tables 3,4 , and 5 . With the monoclonal antibody identifying the HLA class I determinants (2A1) considerable staining was seen in the acini, ducts, and lymphocytes of the patients with both primary and secondary Sjögren's syndrome (Fig. 1). There were few infiltrating lymphocytes seen in the biopsy specimens from the healthy controls, but some staining of the ducts and acini was found, though this was clearly less than that found in patients with Sjögren's syndrome (Fig. 2).

UCHT1 was used to ascertain the presence of $T$ lymphocytes and confirmed that focal collections of these cells were frequently present in the biopsy specimens from patients with secondary Sjögren's syndrome. In contrast, patients with primary Sjögren's syndrome tended to have diffusely scattered $T$ lymphocytes. $T$ lymphocytes were rarely identified in the normal controls.

Although the presence of class II antigens in the ducts and acini varied in both intensity and distribution in different patients, most of the lymphocytes were stained in all patients, indicating that these cells were activated (Fig. 3). At least $60 \%$ of cells in the lymphocyte infiltrates in all patients were stained. Strong $(+++)$ staining was seen in $4 / 8$ patients with primary Sjögren's syndrome, and in 6/8 patients with secondary Sjögren's syndrome with SLE. In the remainder of the biopsy specimens from patients with secondary Sjögren's syndrome (and SLE) at least ++ staining of lymphocytes was seen.

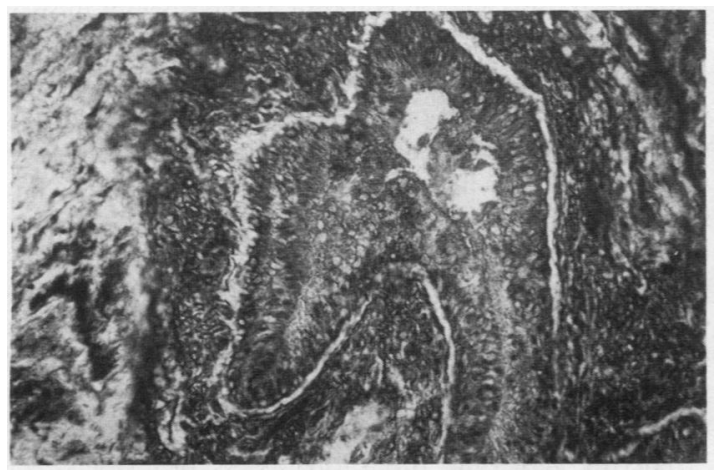

Fig. 1 Patient with secondary Sjogren's syndrome stained for HLA class I using the APAAP technique counterstained with haematoxylin. Most of the cells in the duct and surrounding infiltrate are strongly positive.

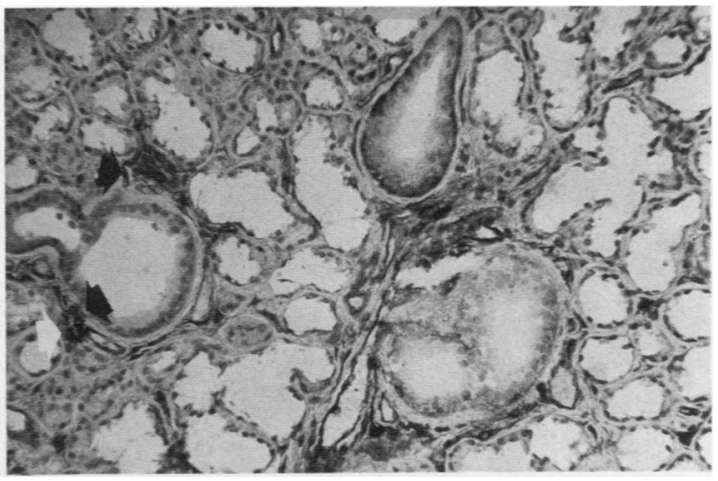

Fig. 2 Normal control stained for HLA class I using the APAAP technique counterstained with haematoxylin. Some positive cells are arrowed. The ducts and acini are predominantly negative.

Overall, there was much less staining with HLA class II (DA2) in the three normal controls, though some ductal and acinar cells were found to be clearly positive in each case (Fig. 4).

The monoclonal antibody identifying interferon- $\alpha$ showed some strong staining $(+++)$ in two of the patients with primary Sjögren's syndrome. This staining was confined largely to the acini. Much less staining (maximum +) was seen in the acini and ducts of the patients with secondary Sjögren's syndrome with SLE. On plasma cells in two patients with secondary Sjögren's syndrome, however, grade ++ was recorded. In the normal controls no staining was seen.

There was little evidence of interferon- $\beta$, with the exception of one patient with primary Sjögren's

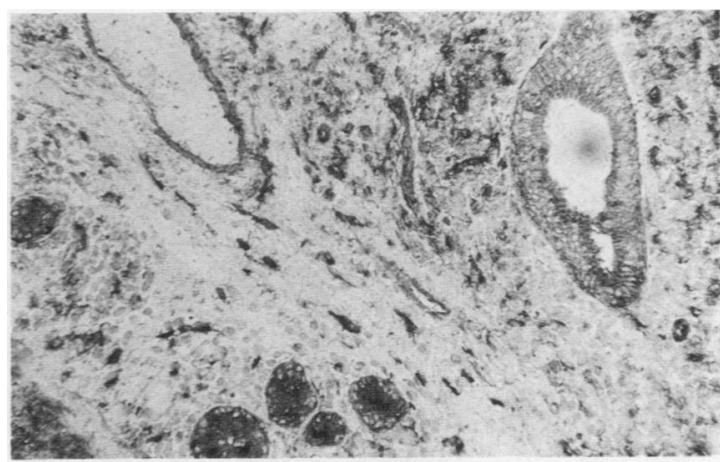

Fig. 3 Patient with secondary Sjögren's syndrome stained for HLA class II using the APAAP technique counterstained with haematoxylin. Ducts and acini are both positively stained. 


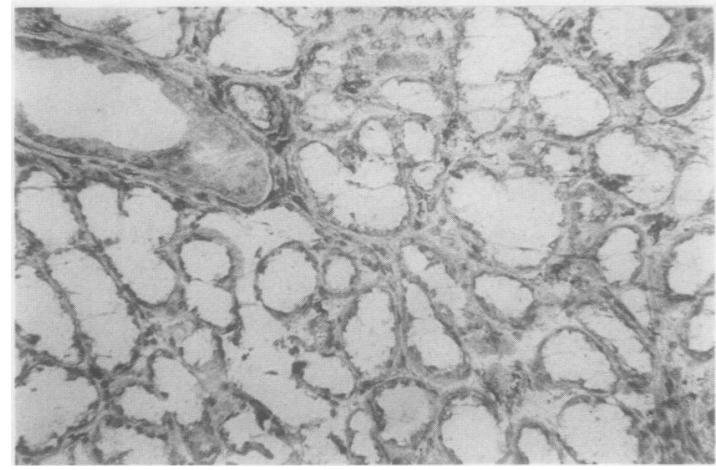

Fig. 4 Normal control stained for HLA class II using the APAAP technique counterstained with haematoxylin. Only a few isolated cells are stained.

syndrome in whom epithelial cells in some ducts were strongly stained. It was negative on all the normal controls.

The monoclonal antibody identifying interferon- $\gamma$ showed a notable pattern of ductal staining. In two of the patients with primary Sjögren`s syndrome. ductal epithelial staining was identified, whereas secretory cells were specifically stained in two of the remaining patients. Among the patients with secondary Sjögren`s syndrome (and SLE) strong ductal staining was seen in three patients and most. if not all, of the ducts were stained (Fig. 5). The staining was only confined to secretory cells in one patient. In two out of three of the normal controls no ductal staining was seen. In one. isolated positive ductal epithelial cells within ducts were seen but not the positive secretory population seen in the patients

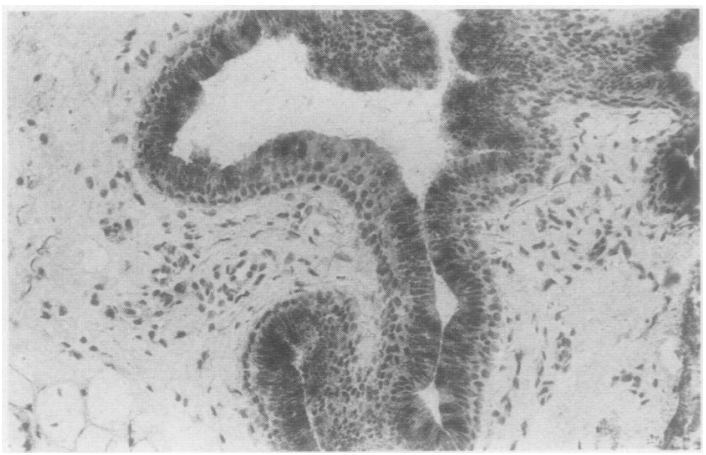

Fig. 5 Patient with secondary Sjögren's syndrome stained for interferon- $\gamma$ using the APAAP technique counterstained with haematoxylin. Strongly stained cells can be seen at intervals in the duct.

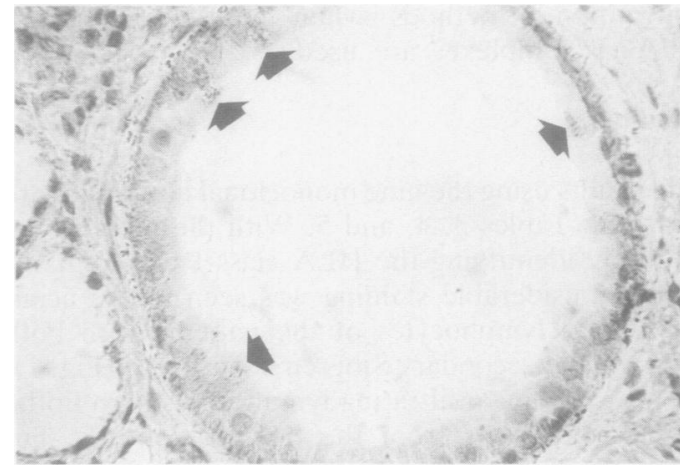

Fig. 6 Normal control stained for interferon-y using the APAAP technique counterstained with haematoxylin. A few cells in the duct (arrowed) are weakly stained.

with Sjögren's syndrome (Fig. 6). This individua had a periductal lymphocytic infiltrate, although the was not surrounding the ducts in which stained cella were seen.

Anti-IL2 (DMS2) showed very little staining Very weak ductal staining, not exceeding + . wä seen in one patient with primary Sjögren's syndroms and in one patient with secondary Sjögren`s syn drome. Focal acinar staining was also seen in ond patient with primary Sjögren's syndrome. Som very weak staining of plasma cells was seen in on? patient with secondary Sjögren's syndrome. Ur identified spindle shaped cells were also stained i⿱ another patient with secondary Sjögren's syndromeg No staining was seen in the normal controls, except for isolated focal deposits previously reported.

The other anti-IL2 antibody, DMS3, showed on extremely weak staining on a few patients, this being. mainly in the acini with ductal staining on twi patients. There was no staining of the normal biops specimens.

Very little staining was seen with anti- $T$ a (CD25). One of the patients with primary Sjögrento syndrome showed strong staining of one acinus, th significance of which is unclear. Weak staining o some ducts and acini was seen on another patienN but this showed weak generalised staining and waN probably not significant. In the patients with secon:dary Sjögren's syndrome, weak staining was seen of macrophages and ducts of one patient and very weak staining on ducts and lymphocytes of anothe No other staining was seen in normal controls.

Having assessed the biopsies as described, reanalysed serial sections from four patients taken $\mathbb{Q}$ random and the controls, to try to correlate tae expression of the class I and II antigens wiff interferon- $\gamma$. Compared with the normals. HLA 
class I antigens were much more strongly expressed on the acini, ducts, and infiltrating lymphocytes. There was a similar increase in HLA class II expression on the acini and lymphocytes, but the increase in duct staining was far more variable. In contrast, increased interferon- $\gamma$ expression was most marked in the ducts, rather less on the acini, and little increased on the lymphocytes.

\section{Discussion}

Evidence has recently accumulated which emphasises the potential role of class II MHC molecules in the initiation of autoimmune phenomena. For example, Bottazzo and colleagues have shown that interferon- $\gamma$ can induce the expression of class II molecules on B lymphocytes, macrophages, and a variety of tissues which do not usually express these molecules. ${ }^{12} 13$ These tissues include thyroid epithelial cells, bile duct epithelium, synovial cells, and islet cells. The co-expression of these HLA D/DR determinants together with other self molecules on the surface of a given tissue may activate adjacent $T$ lymphocytes and induce an autoimmune process.

In this study we have examined tissue from labial glands which bear the brunt of the autoimmune process in Sjögren's syndrome. We and others have shown that many of the infiltrating cells detectable in labial biopsy specimens are $\mathrm{T}$ lymphocytes, predominantly those carrying the helper/inducer phenotype, and that many also express HLA class II antigens, suggesting that they are in the activated state. ${ }^{1} 21+15$ In the study by Fox et al it was claimed that no HLA class II determinants were present on epithelial cells from 'normal subjects'. ${ }^{15}$ We have seen staining of a few acini, but this is much less marked than in the biopsy specimens from the patients with Sjögren's syndrome. This relatively minor discrepancy may be due to their use of necropsy material and tissue from 'histologically normal margins of glands removed because of tumours' to form their normal control material. In contrast, our normal tissue was taken from healthy, living volunteers.

The presence of lymphocytic infiltrates in labial biopsy specimens is not disease specific. Lindahl and colleagues have recently shown that eight of 11 labial biopsy specimens from patients with myasthenia gravis had focal lymphocytic infiltrates with similar helper/inducer phenotypic predominance. ${ }^{16}$ It is noteworthy that despite the infiltrates these patients had a paucity of the usual sicca complex symptomatology, implying that lymphocytic infiltration cannot be the only cause of impaired exocrine glandular dysfunction. This suggestion is supported by our observation that one of our healthy controls had a lymphocytic infiltrate.
We were interested to examine the relation between the HLA class I and II expression with interferon and, IL2 and the IL2 receptor detection. In our studies of polymyositis we were not able to detect HLA class I antigens in normal muscle. ${ }^{5}$ It was found only in areas of myofibre damage or along the sarcolemma/basement membrane of normal looking fibres which were adjacent to the inflammatory infiltrate. Furthermore, interferon- $\alpha$, $\beta$, and $\gamma$ expression was closely matched to that of the HLA class I antigens. ${ }^{6}$

In labial biopsy specimens from the healthy controls there was evidence of both HLA class I and class II expression in both ducts and acini, though it was less marked than in the specimen from patients with either primary or secondary Sjögren's syndrome. This suggests that the mechanisms of tissue damage may be different in Sjögren's syndrome than in myositis, though increased HLA expression may potentiate other mechanisms.

Analysis of the results with the monoclonal antibodies identifying the interferons showed some major differences when compared with our studies in polymyositis. Instead of the rather similar patterns that we found in myositis with each of the three interferons, our results in this study showed obvious differences between them. For example, there was very little evidence of any interferon- $\beta$ expression in any of the biopsy specimens. There was generally weak but persistent interferon- $\alpha$ expression in the ducts and acini in both primary and secondary Sjögren's syndrome, though this was more obvious in the former. Interferon- $\gamma$ staining in the ducts was very striking, especially in the patients with primary Sjögren's syndrome. This evidence lends some support to the view expressed by Fox $e t$ $a l$ that interferon- $\gamma$ is produced locally and induces class II determinants. ${ }^{15}$ Anatomically, however, it is difficult to be certain that the same cells showing increased HLA class I and II expression are also those which show increased expression of interferon- $\gamma$. Further studies using double labelling will answer this point.

\section{References}

1 Isenberg D A. Rowe D. Tookman A. et al. An immunohistological study of secondary Sjögren’s syndrome. Ann Rheum Dis 1984; 43: 470-6.

2 Moutsopoulos H M. Hooks J J, Chan C C, Dalavanga Y A. Skopouli F N. Detrick B. HLA-DR expression by labial minor salivary gland tissues in Sjögren's syndrome. Ann Rheum Dis 1986; 45: 677-83.

3 Fox R I, Carstens S A. Fong S, Robinson C A. Howell F, Vaughan $J H$. Use of monoclonal antibodies to analyze peripheral blood and salivary gland lymphocyte subsets in Sjögren's syndrome. Arthritis Rheum 1982: 25: 419-26.

4 Rowe D J. Isenberg D A. McDougall J, Beverley P C L. 
Characterization of polymyositis infiltrates using monoclonal antibodies to human leucocyte antigens. Clin Exp Immunol 1981: 45: 290-8

5 Rowe D. Isenberg D A. Beverley P C L. Monoclonal antibodies to leucocyte antigens in polymyositis and muscular dystrophy. Clin Exp Immunol 1983: 54: 327-36.

6 Isenberg D A. Rowe D. Shearer M. Novick D. Beverley P C L. Localization of interferons and IL-2 in polymyositis and muscular dystrophy. Clin Exp Immunol 1986: 63: 450-8.

7 Brodsky F M. Parham P. Barnstable C J. Crumpton M J. Bodmer W F. Monoclonal antibodies for analysis of the HLA system. Immunol Rev 1979; 47: 536-45.

8 Novick D. Eshhar Z. Rubinstein M. Monoclonal antibodies to interferon and their use for affinity chromatography. $J \mathrm{Im}$ munol 1982; 129: 2244-7.

9 Novick D, Eshhar Z. Gigi O. Marks Z. Revel M. Rubinstein M. Affinity chromatography of human fibroblasts interferon (IFN-B1) by monoclonal antibody columns. J Gen Virol 1983: 64: $905-10$.

10 Novick D. Eshhar Z. Fischer D G, Friedlander J. Rubinstein M. Monoclonal antibodies to human interferon: production. affinity, purification and radioimmunoassay. $E M B O J$ 1983: 2: 1527-30.
11 Leonard W J. Depper J M. Uchivama H A. Smith K A Waldmann T A. Greene W C. A monoclonal antibody thit: appears to recognise the receptor for human T-cell growt factor: partial characterization of the receptor. Nature $198 \mathrm{z}$ 300: $267-9$

12 Bottazzo G F. Dean B M. McNally J M. MacKay E H. Swift G. Gamble D R. In situ characterization of autoimmune phenomena and expression of HLA molecules in the pancread in diabetic insulitis. $N$ Engl $J$ Med 1985: 313: 353-60

13 Bottazzo G F. Pujol-Borrell R. Hanafusa T. Feldmann M. Rolể of aberrant HLA-DR expression and antigen presentation in induction of endocrine autoimmunity. Lancet 1983: ii: 1115-8.

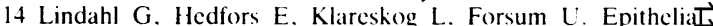
HLA-DR expression in T lymphocyte subsets in salivary glandw in Sjögren's sundrome. Clin Exp Immunol 1985: 61: 475-82

15 Fox RI. Bumol T. Fantozzi R. Bone R. Schrciber Rz Expression of histocompatibility antigen HLA-DR by salivar gland epithelial cells in Sjögren's syndrome. Arthritis Rheu 1986: 29: $11115-11$.

16 Lindahl G. Lefvert A K. Hedfors E. Periductal lymphocvties infiltrates in salivary glands in myasthenia gravis patiento lacking Sjögren's syndrome. (lin Exp lmmunol 1986: $95-1112$ 